(C) The Authors 2015. This is an Open Access article, distributed under the terms of the Creative Commons Attribution licence (http://creativecommons.org/licenses/by/3.0/), which permits unrestricted re-use, distribution, and reproduction in any medium, provided the original work is properly cited.

\title{
Prevalence of hyperhomocysteinaemia and some of its major determinants in Shaanxi Province, China: a cross-sectional study
}

\author{
Xue-Dong $\mathrm{Liu}^{1}$, Bin $\mathrm{GaO}^{2}$, Dong Sun ${ }^{1}$, Ming Shi ${ }^{1}$, Yue-Yun $\mathrm{Ma}^{3}$, Zhi-Rong Liu ${ }^{1}$, Bo Wang ${ }^{4}$, Xiping Xu ${ }^{5}$, \\ $\mathrm{Xin} \mathrm{Xu}^{5}$, Qiu-He $\mathrm{Ji}^{2 *}$ and Gang Zhao ${ }^{1 *}$ \\ ${ }^{1}$ Department of Neurology, Xijing Hospital, The Fourth Military Medical University, Changle Xi Road 17, Xi'an 710032, \\ Shaanxi Province, People's Republic of China \\ ${ }^{2}$ Department of Endocrinology, Xijing Hospital, The Fourth Military Medical University, Changle Xi Road 17, Xi'an 710032, \\ Shaanxi Province, People's Republic of China \\ ${ }^{3}$ Department of Clinical Laboratory, Xijing Hospital, The Fourth Military Medical University, Xi'an, Shaanxi, \\ People's Republic of China \\ ${ }^{4}$ Department of Epidemiology, School of Military Preventive Medicine, The Fourth Military Medical University, Xi'an, \\ Shaanxi, People's Republic of China \\ ${ }^{5}$ Institute of Nephrology, Southern Medical University, Guangzhou, Guangdong, People's Republic of China
}

(Submitted 30 May 2014 - Final revision received 18 November 2014 - Accepted 18 November 2014 - First published online 30 January 2015)

\section{Abstract}

The aim of the present study was to investigate the prevalence of hyperhomocysteinaemia (HHCY; total plasma homocysteine (tHcy) concentration $>15 \mu \mathrm{mol} / \mathrm{l}$ ) and its major determinants in healthy Chinese northerners. A descriptive and cross-sectional study was conducted in Shaanxi Province, China. The study sample included 2645 participants (1042 men and 1603 women) aged $>20$ years. Demographic characteristics and lifestyle factors were assessed via questionnaire interviews and physical examination. Plasma levels of homocysteine and folate and methylenetetrahydrofolate reductase (MTHFR) gene polymorphism were determined according to standard methods. The prevalence of HHCY was $67.7 \%$ ( $81.4 \%$ in men and $58.8 \%$ in women). The geometric mean of tHcy concentration was $19 \cdot 1 \mu \mathrm{mol} / \mathrm{l}$. The OR of HHCY were 0.44 (95\% CI 0.34, 0.57) for women $v$. men; 1.95 (95\% CI 1.41, 2.70$), 1.41$ (95\% CI 1.05, 1.88 ) and 0.76 (95\% CI $0 \cdot 64,0 \cdot 89)$ for participants with smoking and alcohol drinking cessation and improved physical activity levels, respectively; $0 \cdot 25$ ( $95 \%$ CI $0 \cdot 17,0.38), 0.33(95 \%$ CI $0.22,0.49)$ and $0.56(95 \%$ CI $0.36,0.88)$ for participants with an education level of elementary school, secondary school and university $v$. illiterate, respectively; 1.41 (95\% CI 1.13, 1.75) and 3.05 (95\% CI 2.35, 3.97) for participants with CT and TT $v$. CC genotype at MTHFR $677 \mathrm{C} \rightarrow \mathrm{T}$ polymorphism, respectively. These results demonstrate that the prevalence of HHCY is considerably high in Chinese northerners, especially in TT subjects, suggesting that implementation of tHcy-lowering strategies, such as lifestyle changes, is necessary.

\section{Key words: Prevalence: Hyperhomocysteinaemia: Determinants: Chinese northerners}

Homocysteine is a S-containing amino acid generated during the catabolism of the essential amino acid methionine. It is produced by intracellular demethylation of methionine and then exported to the plasma, where it circulates, mainly in its oxidised form. Abundant studies have established elevated plasma total homocysteine (tHcy) as a potent independent risk factor for coronary artery disease, stroke and deep vein thrombosis $^{(1,2)}$.

Elevation in tHcy levels can be caused by one or a combination of genetic, physiological, pathological and nutritional factors. Sex, race/ethnicity, age, smoking status, physical activity and dietary patterns are directly associated with serum tHcy concentrations ${ }^{(3)}$. Folate, cobalamin and pyridoxine are important regulators in homocysteine metabolism. Furthermore, studies have shown an inverse relationship between folate intake and homocysteine levels ${ }^{(4,5)}$. The complementary DNA of cystathione- $\beta$-synthase, methylenetetrahydrofolate reductase (MTHFR), methionine synthase and methionine synthase reductase have all been cloned and analysed for functional polymorphisms that affect homocysteine/ folate metabolism. The MTHFR C677T mutation is the most frequent cause of moderate hyperhomocysteinaemia (HHCY)

Abbreviations: HHCY, hyperhomocysteinaemia; MTHFR, methylenetetrahydrofolate reductase; tHcy, total homocysteine.

*Corresponding authors: Q.-H. Ji, email jqiuhe@fmmu.edu.cn; G. Zhao, email zhaogang@fmmu.edu.cn 
due to genetic factors, especially in individuals with low folate status $^{(6)}$.

Developed countries have carried out several large-scale population-based surveys to evaluate plasma tHcy status and its determinants ${ }^{(7)}$; however, there is a paucity of equivalent data from developing countries. China is vast in territory and has fifty-six minorities. The levels of tHcy vary greatly with region and nation. Although studies have reported the distribution of plasma tHcy levels in some Chinese populations ${ }^{(8,9)}$, they were limited by sampling issue and small sample sizes. The main purpose of the present study was to investigate the prevalence of HHCY and its major determinants in the general population of northern China.

\section{Subjects and methods}

\section{Study population}

A descriptive, cross-sectional epidemiological study was conducted to estimate plasma homocysteine levels in the general population of Shaanxi Province. Shaanxi is a province located in northern China, with an area of $205800 \mathrm{~km}^{2}$ and a population of 40000000 . The subjects of the present analysis were part of a larger study, namely the China National Diabetes and Metabolic Disorders Study (CNDMDS). The original CNDMDS cohort ( $n$ 46239) was recruited by a multi-stage, stratified sampling method at baseline (2007-8) from fourteen provinces and municipalities in China to estimate the prevalence of diabetes among Chinese adults. The design and results of the study have been described in detail elsewhere $^{(10)}$. In the present study, we included subjects aged 20 years or older from Shaanxi Province. Only subjects who had lived in their current residence for 5 years or longer were eligible to participate. The participants came from urban street districts and rural villages that would ensure representation of socio-economic differences. Subjects with the following conditions were excluded from the study: those who were unwilling to participate in the survey; those who presented with some types of acute or chronic illnesses such as cancer and severe renal and liver diseases.

\section{Data collection procedures}

Baseline data were collected by trained research staff according to the standard operating procedure. Each participant was interviewed using a standard questionnaire to obtain information on demographic characteristics, personal and medical history, and lifestyle risk factors.

Behaviour. Cigarette smoking, alcohol drinking and physical activity were defined according to a previous study ${ }^{(10)}$. In brief, cigarette smoking referred to having smoked at least 100 cigarettes in one's lifetime. Alcohol drinking referred to consumption of at least $30 \mathrm{~g}$ of alcohol/week for 1 year or more. Physical activity referred to participation in moderate or vigorous activity for $30 \mathrm{~min}$ or more per $\mathrm{d}$ for at least $3 \mathrm{~d}$ per week. According to the Chinese educational system, educational levels were grouped according to self-reported years in school ( $0-6$ years, illiterate; 7-12 years, elementary school; 13-16 years, secondary school; $>16$ years, university).

Anthropometric measurements. Height, weight, and waist and hip circumference were taken using the standard operating procedure. Height was measured without shoes to the nearest $0.5 \mathrm{~cm}$ using a portable stadiometer. Weight was measured in light indoor clothing without shoes to the nearest $0.5 \mathrm{~kg}$. BMI was calculated as weight $(\mathrm{kg}) /$ height $\left(\mathrm{m}^{2}\right)$. Waist circumference was measured at the level of the umbilicus without clothing and in standing position. Hip circumference was measured at the level of ischial tuberosity. The waist:hip ratio was calculated as waist/hip.

Blood pressure. Seated blood pressure measurements were obtained by trained research staff after subjects had been seated for $10 \mathrm{~min}$ using a $\mathrm{Hg}$ manometer, and using the standard method of calibration and appropriately sized cuffs, according to the standard operating procedure. Triplicate measurements were taken on the same arm, with an interval of at least $2 \mathrm{~min}$. Systolic and diastolic blood pressure of each patient were calculated as the mean of three independent measures.

All study investigators and staff members received a complete training programme, including the usage of the questionnaire and data collection, according to a standard protocol.

\section{Blood sample collection and laboratory methods}

After $12-15 \mathrm{~h}$ of fasting, a $15 \mathrm{ml}$ venous blood sample was collected from each subject by venepuncture using a Vacutainer tube containing EDTA-Na $\mathrm{Na}_{2}$ as an anti-coagulant. Whole blood was centrifuged at $3000 \mathrm{rpm}$ for $10 \mathrm{~min}$. Plasma samples were separated and transferred on dry ice to the central laboratory of Xijing Hospital, Shaanxi Province. Glucose, cholesterol and TAG levels were assessed enzymatically using commercially available reagents. The remaining plasma samples were stored at $-70^{\circ} \mathrm{C}$ (Thermo) for 8 months to 2 years before being analysed for tHcy and folate concentrations.

Homocysteine concentration was determined by an enzymatic assay ${ }^{(11)}$ using the Hitachi 7020 Automatic Analyzer. The method has been validated over a linearity range of 1-65 $\mu \mathrm{mol} / 1$ from plasma. Plasma folate concentrations were determined by a microbial assay (Lactobacillus casei) using a ninety-six-well plate ${ }^{(12)}$. The MTHFR $677 \mathrm{C} \rightarrow \mathrm{T}$ polymorphism was determined by the TaqMan assay designed and manufactured by Applied Biosystems. The intra-assay and inter-assay $\mathrm{CV}$ were $5 \cdot 1$ and $6 \cdot 9 \%$ for homocysteine and $6 \cdot 2$ and $7 \cdot 1 \%$ for folate, respectively. All laboratory test methods were certified by the National Health and Family Planning Commission of China. Although epidemiological surveys have determined tHcy concentrations in order to identify the prevalence of HHCY, estimation of these cases is complicated by the lack of a standard definition of high tHcy concentration. Different thresholds for HHCY, more or less arbitrary, have been used in most studies, such as values above the mean found in the control group plus two standard deviations, $>95$ th percentile, $>10 \mu \mathrm{mol} / \mathrm{l}^{(8)},>13 \mu \mathrm{mol} / \mathrm{l}^{(13)}$ and $>15 \mu \mathrm{mol} / \mathrm{l}^{(14)}$. In the present study, individuals were 
Table 1. Characteristics of the study population by sex in Shaanxi Province, China*

(Number of participants and percentages; mean values and standard deviations)

\begin{tabular}{|c|c|c|c|c|c|c|c|}
\hline \multirow[b]{2}{*}{ Characteristics } & \multicolumn{2}{|c|}{ All (n 2645) } & \multicolumn{2}{|c|}{ Men ( $n$ 1042) } & \multicolumn{2}{|c|}{ Women (n 1603) } & \multirow[b]{2}{*}{$P \dagger$} \\
\hline & $n$ & $\%$ & $n$ & $\%$ & $n$ & $\%$ & \\
\hline Age (years) & & & & & & & 0.896 \\
\hline Mean & \multicolumn{2}{|c|}{43.57} & \multicolumn{2}{|c|}{43.52} & \multicolumn{2}{|c|}{43.59} & \\
\hline $\mathrm{SD}$ & \multicolumn{2}{|c|}{13.49} & \multicolumn{2}{|c|}{13.52} & \multicolumn{2}{|c|}{13.47} & \\
\hline Height (cm) & & & & & & & $<0.001$ \\
\hline Mean & & & & & & & \\
\hline SD & & & & & & & \\
\hline Weight (kg) & & & & & & & $<0.001$ \\
\hline Mean & & & & & & & \\
\hline SD & & & & & & & \\
\hline BMI $\left(\mathrm{kg} / \mathrm{m}^{2}\right)$ & & & & & & & $<0.001$ \\
\hline Mean & & & & & & & \\
\hline SD & & & & & & & \\
\hline WHR & & & & & & & $<0.001$ \\
\hline Mean & & & & & & & \\
\hline SD & & & & & & & \\
\hline Education & & & & & & & $<0.001$ \\
\hline University & 700 & $26 \cdot 5$ & 330 & $31 \cdot 7$ & 373 & $23 \cdot 3$ & \\
\hline Secondary school & 1310 & 49.5 & 548 & 52.6 & 777 & 48.5 & \\
\hline Elementary school & 417 & $15 \cdot 8$ & 127 & $12 \cdot 2$ & 288 & $18 \cdot 0$ & \\
\hline Illiterate & 218 & $8 \cdot 2$ & 37 & 3.5 & 165 & $10 \cdot 3$ & \\
\hline Smoking & 595 & 22.5 & 575 & $55 \cdot 2$ & 20 & $1 \cdot 2$ & $<0.001$ \\
\hline Alcohol drinking & 600 & $22 \cdot 7$ & 521 & $50 \cdot 0$ & 78 & 4.9 & $<0.001$ \\
\hline Physical activity & 929 & $35 \cdot 1$ & 380 & $36 \cdot 5$ & 553 & 34.5 & 0.558 \\
\hline History of hypertension & 390 & $14 \cdot 7$ & 139 & $13 \cdot 3$ & 251 & $15 \cdot 7$ & 0.206 \\
\hline History of hyperlipaemia & 178 & $6 \cdot 7$ & 88 & $8 \cdot 4$ & 90 & $5 \cdot 6$ & 0.012 \\
\hline History of hypercholesterolaemia & 79 & 2.9 & 33 & $3 \cdot 2$ & 46 & 2.9 & 0.681 \\
\hline $\mathrm{SBP}(\mathrm{mmHg})$ & & & & & & & $<0.001$ \\
\hline Mean & & & & & & & \\
\hline SD & & & & & & & \\
\hline $\mathrm{DBP}(\mathrm{mmHg})$ & & & & & & & $<0.001$ \\
\hline Mean & & & & & & & \\
\hline SD & & & & & & & \\
\hline Glucose (mmol/l) & & & & & & & 0.74 \\
\hline Mean & & & & & & & \\
\hline SD & & & & & & & \\
\hline Total cholesterol (mmol/l) & & & & & & & 0.96 \\
\hline Mean & & & & & & & \\
\hline SD & & & & & & & \\
\hline TAG (mmol/l) & & & & & & & $<0.001$ \\
\hline Mean & & & & & & & \\
\hline SD & & & & & & & \\
\hline HDL-C (mmol/l) & & & & & & & $<0.001$ \\
\hline Mean & & & & & & & \\
\hline SD & & & & & & & \\
\hline LDL-C (mmol/l) & & & & & & & 0.023 \\
\hline Mean & & & & & & & \\
\hline SD & & & & & & & \\
\hline Homocysteine $(\mu \mathrm{mol} / \mathrm{l})$ & & & & & & & $<0.001$ \\
\hline Geometric mean & & & & & & & \\
\hline SD & & & & & & & \\
\hline Homocysteine group & & & & & & & $<0.001$ \\
\hline$<15 \mu \mathrm{mol} / \mathrm{l}$ & 854 & $32 \cdot 3$ & 194 & $18 \cdot 6$ & 660 & $41 \cdot 2$ & \\
\hline $15-30 \mu \mathrm{mol} / \mathrm{l}$ & 1260 & $47 \cdot 6$ & 540 & $51 \cdot 8$ & 720 & 44.9 & \\
\hline $30-65 \mu \mathrm{mol} / \mathrm{l}$ & 454 & $17 \cdot 2$ & 249 & 23.9 & 205 & $12 \cdot 8$ & \\
\hline$\geq 65 \mu \mathrm{mol} / /$ & 77 & 2.9 & 59 & $5 \cdot 7$ & 18 & $1 \cdot 1$ & \\
\hline MTHFR $677 \mathrm{C} \rightarrow$ T polymorphism & & & & & & & 0.398 \\
\hline TT & 725 & $27 \cdot 4$ & 271 & $26 \cdot 0$ & 454 & $28 \cdot 3$ & \\
\hline $\mathrm{CC}$ & 597 & $22 \cdot 6$ & 236 & $22 \cdot 6$ & 361 & 22.5 & \\
\hline $\mathrm{CT}$ & 1323 & $50 \cdot 0$ & 535 & $51 \cdot 3$ & 788 & $49 \cdot 2$ & \\
\hline Plasma folate $(\mathrm{nmol} / \mathrm{l})$ & & & & & & & $<0.001$ \\
\hline Geometric mean & & & & & & & \\
\hline SD & & & & & & & \\
\hline Plasma folate group & & & & $<0.001$ & & & \\
\hline$<3.4 \mathrm{nmol} / \mathrm{l}$ & 378 & $14 \cdot 3$ & 173 & $16 \cdot 6$ & 205 & $12 \cdot 8$ & \\
\hline $3.4-6.8 \mathrm{nmol} / \mathrm{l}$ & 1045 & 39.5 & 443 & $42 \cdot 5$ & 602 & 37.6 & \\
\hline$>6.8 \mathrm{nmol} / \mathrm{l}$ & 1222 & $46 \cdot 2$ & 426 & 40.9 & 796 & $49 \cdot 6$ & \\
\hline
\end{tabular}

WHR, waist:hip ratio; SBP, systolic blood pressure; DBP, diastolic blood pressure; HDL-C, HDL-cholesterol; LDL-C, LDL-cholesterol; MTHFR, methylenetetrahydrofolate reductase.

*Because plasma total homocysteine and folate concentrations were not normally distributed, natural logarithmic transformation was used to satisfy the normality requirement. $\dagger P$ values were obtained by $t$ test for comparing quantitative variables and $\chi^{2}$ test for comparing qualitative variables between men and women. 
classified as having HHCY if they had tHcy concentrations $>15 \mu \mathrm{mol} / \mathrm{l}$. Individuals were classified as having plasma folate deficiency if they had plasma folate concentrations $<6.8 \mathrm{nmol} / \mathrm{l}^{(15)}$.

\section{Statistical analyses}

For a positively skewed distribution of plasma tHcy and plasma folate concentrations, natural logarithmic transformation was used to obtain better normality. Differences in population characteristics by sex were compared using Student's $t$ tests or $\chi^{2}$ test. Differences in homocysteine and folate levels by the MTHFR $677 \mathrm{C} \rightarrow \mathrm{T}$ polymorphism were compared using ANOVA or $\chi^{2}$ test.

Spearman's correlation analysis was performed to assess the association of plasma homocysteine levels with folate and quantitative anthropometric, biochemical and metabolic variables. HHCY was examined using a multivariate logistic regression model with independent variables including age group (20-40, 41-60 and $>60$ years), sex, BMI $(<25 v$. $\geq 25 \mathrm{~kg} / \mathrm{m}^{2}$ ), education level (illiterate, elementary school, secondary school and university), smoking status, alcohol drinking, physical activity, MTHFR $677 \mathrm{C} \rightarrow \mathrm{T}$ polymorphism (CC, CT and TT), systolic blood pressure $(<140 \quad v$. $\geq 140 \mathrm{mmHg}$ ), diastolic blood pressure $(<90 \mathrm{v}$. $\geq 90 \mathrm{mmHg})$, TAG concentration $(\leq 1.80 v .>1.80 \mathrm{mmol} / \mathrm{l})$, HDL-cholesterol concentration $(<1.68 v$. $\geq 1.68 \mathrm{mmol} / \mathrm{l})$ and folate concentration $(<6.8 v$. $\geq 6.8 \mathrm{nmol} / \mathrm{l})$. We also investigated possible interactions among the genotype, plasma folate and sex by including an interaction term in the regression model. We assessed goodness of fit by the Hosmer-Lemeshow test. The level of significance was set at $P<0.05$.

All statistical analyses were performed using the SPSS 11.0 statistical package (SPSS, Inc.).

\section{Ethical approval}

The present study was conducted according to the guidelines laid down in the Declaration of Helsinki, and all procedures involving human subjects were approved by the Ethics Committee of the Institute of Xijing Hospital, Fourth Military Medical University, Shaanxi, China. Written informed consent was obtained from all subjects. For illiterate subjects, data collectors read the consent paper to them, and their fingerprints were taken if they agreed.

\section{Results}

A total of 3297 subjects were selected and invited to participate in the present study. Of these, 237 were unwilling to take part in the study, 108 presented with some types of acute or chronic illness, and 307 had missing data on age, height, weight, BMI, smoking status, alcohol drinking, standard of education, physical activity, total cholesterol, TAG, HDL-cholesterol, LDL-cholesterol, plasma tHcy, folate or MTHFR $\quad 677 \mathrm{C} \rightarrow \mathrm{T}$ polymorphism; therefore, they were excluded from the study. A final sample of 2645 subjects (1042 men and 1603 women) was included in the analysis.

The characteristics of the study population by sex are summarised in Table 1. Men had significantly higher values for height, weight, BMI, waist:hip ratio, education level, percentage of cigarette smoking and alcohol drinking, history of hyperlipaemia, systolic blood pressure, diastolic blood pressure, and TAG, LDL-cholesterol and homocysteine geometric mean, and had lower values for HDL-cholesterol and folate geometric mean.

The prevalence of HHCY (tHcy concentrations $>15 \mu \mathrm{mol} / \mathrm{l}$ ) was $67.7 \%$ ( $81.4 \%$ in men and $58.8 \%$ in women). tHcy levels were $>30 \mu \mathrm{mol} / 1$ in $20.1 \%$ of adults (29.6\% in men and $13.9 \%$ in women). The prevalence of HHCY was shifted towards higher levels for men than for women. The geometric mean of homocysteine concentration in the total sample was $19 \cdot 01 \mu \mathrm{mol} / \mathrm{l}$ (men $23.45 \mu \mathrm{mol} / \mathrm{l}$; women $16.58 \mu \mathrm{mol} / \mathrm{l}$ ). When analysing the MTHFR $677 \mathrm{C} \rightarrow \mathrm{T}$ polymorphism, the normal homozygous CC genotype was present in $22.6 \%$ of cases, the heterozygous CT genotype was present in $50 \%$ of cases, and the TT genotype, corresponding to homozygous mutation, was present in $27.4 \%$ of cases.

The prevalence of HHCY, low folate concentrations and MTHFR $677 \mathrm{C} \rightarrow \mathrm{T}$ polymorphism frequencies, stratified by sex and age group, is summarised in Table 2. The age-related increase in tHcy concentrations was observed only in women $(P<0 \cdot 01)$. The prevalence of low folate concentrations and MTHFR $677 \mathrm{C} \rightarrow \mathrm{T}$ polymorphism did not differ by age group.

Homocysteine and folate levels according to MTHFR $677 \mathrm{C} \rightarrow \mathrm{T}$ polymorphism are presented in Table 3. Subjects with the TT genotype had significantly higher tHcy levels

Table 2. Prevalence of hyperhomocysteinaemia (HHCY), low folate concentrations and methylenetetrahydrofolate reductase (MTHFR) $677 \mathrm{C} \rightarrow$ T polymorphism according to sex and age group

\begin{tabular}{|c|c|c|c|c|c|c|c|}
\hline \multirow[b]{2}{*}{ Sex } & \multirow{2}{*}{$\begin{array}{l}\text { Age group } \\
\text { (years) }\end{array}$} & \multirow[b]{2}{*}{$n$} & \multirow{2}{*}{$\begin{array}{c}\mathrm{HHCY} \\
(\%)\end{array}$} & \multirow{2}{*}{$\begin{array}{c}\text { Low } \\
\text { folate }(\%)\end{array}$} & \multicolumn{3}{|c|}{$\begin{array}{l}\text { MTHFR } 677 \mathrm{C} \rightarrow \mathrm{T} \\
\text { polymorphism }\end{array}$} \\
\hline & & & & & TT & $\mathrm{CC}$ & CT \\
\hline \multirow[t]{3}{*}{ Male } & $20-40$ & 464 & $81 \cdot 2$ & 58.6 & $25 \cdot 9$ & 23.1 & 51.0 \\
\hline & $40-60$ & 433 & $83 \cdot 1$ & $59 \cdot 6$ & $25 \cdot 2$ & 23.8 & 51.0 \\
\hline & $>60$ & 145 & 76.6 & $60 \cdot 0$ & 29.0 & 17.9 & 53.1 \\
\hline \multirow[t]{3}{*}{ Female } & $20-40$ & 694 & $50 \cdot 6^{\star \star}$ & $51 \cdot 3$ & $26 \cdot 9$ & 23.5 & 49.6 \\
\hline & $40-60$ & 702 & 63.5 & 49.9 & $30 \cdot 1$ & 22.8 & $47 \cdot 1$ \\
\hline & $>60$ & 207 & 70.5 & $49 \cdot 3$ & $27 \cdot 1$ & 18.4 & 54.6 \\
\hline
\end{tabular}

** Significant at $P<0.01$. 
Table 3. Homocysteine and folate levels according to methylenetetrahydrofolate reductase (MTHFR) $677 \mathrm{C} \rightarrow$ T polymorphism*

(Number of participants and percentages; mean values and standard deviations)

\begin{tabular}{|c|c|c|c|c|c|c|c|}
\hline \multirow[b]{3}{*}{ Characteristics } & \multicolumn{6}{|c|}{ MTHFR $677 \mathrm{C} \rightarrow$ T polymorphism } & \multirow[b]{3}{*}{$P \dagger$} \\
\hline & \multicolumn{2}{|c|}{ TT $(n 725)$} & \multicolumn{2}{|c|}{$\mathrm{CC}(n 597)$} & \multicolumn{2}{|c|}{ CT $(n$ 1323) } & \\
\hline & $n$ & $\%$ & $n$ & $\%$ & $n$ & $\%$ & \\
\hline Homocysteine $(\mu \mathrm{mol} / \mathrm{l})$ & \multirow{2}{*}{\multicolumn{2}{|c|}{23.99}} & & & & & $<0.001$ \\
\hline Geometric mean & & & \multicolumn{2}{|c|}{16.98} & \multicolumn{2}{|c|}{17.78} & \\
\hline SD & \multicolumn{2}{|c|}{1.99} & \multicolumn{2}{|c|}{1.69} & \multicolumn{2}{|c|}{1.74} & \\
\hline \multicolumn{7}{|l|}{ Homocysteine group } & $<0.001$ \\
\hline$<15 \mu \mathrm{mol} / \mathrm{l}$ & 160 & $22 \cdot 1$ & 241 & $40 \cdot 4$ & 453 & 34.2 & \\
\hline $15-30 \mu \mathrm{mol} / \mathrm{l}$ & 292 & $40 \cdot 3$ & 287 & 48.1 & 681 & 51.5 & \\
\hline $30-65 \mu \mathrm{mol} / \mathrm{l}$ & 225 & 31.0 & 60 & $10 \cdot 1$ & 169 & $12 \cdot 8$ & \\
\hline$\geq 65 \mu \mathrm{mol} / \mathrm{l}$ & 48 & $6 \cdot 6$ & 9 & 1.5 & 20 & 1.5 & \\
\hline \multicolumn{7}{|l|}{ Plasma folate $(\mathrm{nmol} / \mathrm{l})$} & $<0.001$ \\
\hline Geometric mean & \multicolumn{2}{|c|}{$5 \cdot 01$} & \multicolumn{2}{|c|}{$6 \cdot 92$} & \multicolumn{2}{|c|}{$6 \cdot 46$} & \\
\hline SD & \multicolumn{2}{|c|}{1.77} & \multicolumn{2}{|c|}{1.66} & \multicolumn{2}{|c|}{1.74} & \\
\hline Plasma folate group & & & & & & & $<0.001$ \\
\hline$<3.4 \mathrm{nmol} / \mathrm{l}$ & 160 & $22 \cdot 1$ & 55 & $9 \cdot 2$ & 163 & $12 \cdot 3$ & \\
\hline $3.4-6.8 \mathrm{nmol} / \mathrm{l}$ & 339 & 46.8 & 207 & 34.7 & 499 & 37.7 & \\
\hline$>6.8 \mathrm{nmol} / \mathrm{l}$ & 226 & 31.2 & 335 & 56.1 & 61 & 50.0 & \\
\hline
\end{tabular}

*Because plasma total homocysteine and folate concentrations were not normally distributed, natural logarithmic transformation was used to satisfy the normality requirement.

$\dagger P$ values were obtained using ANOVA for comparing quantitative variables and using $\chi^{2}$ test for comparing qualitative variables among the MTHFR $677 \mathrm{C} \rightarrow$ T polymorphism

and lower folate levels than those with the CT or CC genotype $(P<0.001)$. The prevalence of HHCY and low folate levels in TT subjects was also higher than that in CT or CC subjects $(P<0 \cdot 001)$.

We estimated the correlation between tHcy levels and common CVD risk factors. Weak positive correlations were observed with age $(r$ 0.095; $P<0.001)$, BMI ( $r$ 0.099; $P<0.001)$, waist:hip ratio $(r 0.160 ; P<0.001)$, diastolic blood pressure $(r$ 0.168; $P<0.001)$ and TAG $(r 0.105 ; P<0.001)$, and negative correlations with folate $(r-0.116 ; P<0.001)$ and HDL-cholesterol $(r-0 \cdot 136 ; P<0 \cdot 001)$.

The results from logistic regression analyses for HHCY are presented in Table 4. In general, smoking status, alcohol drinking, lower education level and T-allele of MTHFR
$677 \mathrm{C} \rightarrow \mathrm{T}$ were associated with an increased risk of HHCY, whereas sex (women) and physical activity level were associated with decreased risk. The genotype $\times$ folate interaction in the regression model was not significant $(P>0.05)$.

\section{Discussion}

In the present study, the geometric mean of tHcy concentrations was $19.01 \mu \mathrm{mol} / 1$. The prevalence of HHCY and folate deficiency was $67.7 \%$ (58.8\% in women and $81.4 \%$ in men) and $53.9 \%(50.4 \%$ in women and $59.2 \%$ in men), respectively.

In developed countries, elevated plasma homocysteine levels are rather infrequent. In Chinese populations, the

Table 4. Multivariate logistic regression analysis for hyperhomocysteinaemia*

(Number of participants and percentages; adjusted odds ratios and $95 \%$ confidence intervals)

\begin{tabular}{|c|c|c|c|c|c|}
\hline & \multicolumn{2}{|c|}{ Prevalence } & \multirow[b]{2}{*}{ Adjusted OR } & \multirow[b]{2}{*}{$95 \% \mathrm{Cl}$} & \multirow[b]{2}{*}{$P$} \\
\hline & $n$ & $\%$ & & & \\
\hline Sex (women) & 943 & $58 \cdot 8$ & 0.44 & $0.34,0.57$ & 0.000 \\
\hline Smoking & 512 & $86 \cdot 1$ & 1.95 & $1.41,2 \cdot 70$ & 0.000 \\
\hline Alcohol drinking & 484 & 80.7 & 1.41 & $1.05,1.88$ & 0.020 \\
\hline Physical activity & 588 & $63 \cdot 3$ & 0.76 & $0.64,0.89$ & 0.001 \\
\hline \multicolumn{6}{|l|}{ Education } \\
\hline Illiterate & 179 & $82 \cdot 2$ & 1.00 & Ref. & \\
\hline Elementary school & 303 & $72 \cdot 7$ & 0.25 & $0.17,0.38$ & 0.000 \\
\hline Secondary school & 873 & $66 \cdot 6$ & 0.33 & $0.22,0.49$ & 0.000 \\
\hline University & 430 & $61 \cdot 4$ & 0.56 & $0.36,0.88$ & 0.011 \\
\hline \multicolumn{6}{|c|}{ MTHFR $677 \mathrm{C} \rightarrow$ T polymorphism } \\
\hline $\mathrm{CC}$ & 356 & $59 \cdot 6$ & 1.00 & Ref. & \\
\hline CT & 870 & $65 \cdot 8$ & 1.41 & $1 \cdot 13,1.75$ & 0.002 \\
\hline TT & 565 & 77.9 & 3.05 & $2.35,3.97$ & 0.000 \\
\hline
\end{tabular}

Ref., reference; MTHFR, methylenetetrahydrofolate reductase.

${ }^{*}$ Adjusted for sex, smoking status, alcohol drinking, physical activity, education level and MTHFR $677 \mathrm{C} \rightarrow \mathrm{T}$ polymorphism. All variables used in the adjusted model were categorical variables. 
reported prevalence of HHCY ranges from 10 to $56 \%$ with various cut-off values of $\mathrm{tHcy}^{(8,9)}$. Generally, the northerners had higher tHcy levels than the southerners. The present results in Shaanxi Province are notably higher than what other surveys have reported in other northern populations such as Hebei and Shanxi Province ${ }^{(8,9)}$. If HHCY is defined as a tHcy concentration $>10 \mu \mathrm{mol} / 1$, the prevalence of HHCY was found to be $88.9 \%(84.8 \%$ in women and $95.2 \%$ in men). The reported differences in tHcy levels in various studies could be due to geographical variations, racial/ethnic differences, different population inclusion criteria, different genetic backgrounds and different lifestyles in the country. Intakes of green leafy vegetables, fruit and seafood with high folate content were much lower in the northern population than in the southern population, and total alcohol consumption was higher. The most notable finding in the present study was that a high prevalence of HHCY and folate deficiency was also common in the northerners.

The present study found that a higher prevalence of HHCY was associated with men, smoking status, alcohol drinking, less exercise, lower education level and TT genotype of MTHFR $677 \mathrm{C} \rightarrow \mathrm{T}$ polymorphism.

Some of the largest population-based studies such as the Third US National Health and Nutrition Examination Survey $^{(3)}$ have demonstrated that Hcy concentrations were higher in men than in women. The present study is in agreement with these results. This difference in homocysteine concentrations could be due to larger muscle mass and greater creatine phosphate synthesis in men, differences in sex-related hormones and differences in vitamin status ${ }^{(16)}$, and homocysteine formation between sexes. Although the age-related increase in the prevalence of HHCY has only been found in women, this might be due to the low hormone levels caused by menopause, since homocysteine concentration has been found to be higher in postmenopausal women than in pre-menopausal women ${ }^{(17)}$.

Cigarette smoking is associated with increased plasma levels of homocysteine, as described in previous studies ${ }^{(18)}$. It has a dose-dependent relationship between the number of cigarettes smoked per $\mathrm{d}$ and the level of tHcy in all age and sex groups $^{(7)}$. The precise reasons remain unknown. Perhaps smokers had a lower intake of most vitamins and were less likely to have consumed fruit, vegetables, and vitamin and mineral supplements. In the present study, smoking was also a determinant of HHCY.

Previous reports on the relationship between alcohol consumption and tHcy concentrations have been inconclusive. Some studies have shown that tHcy is positively associated with alcohol intake ${ }^{(19,20)}$. However, another study has shown an inverse association between Hcy levels and alcohol consumption $^{(8)}$. The present study also observed that alcohol drinking was independently associated with the prevalence of HHCY. Whether the effect of alcohol drinking on tHcy concentrations depends on the type of alcoholic beverage consumed in this population remains to be determined conclusively. The predominant type of alcoholic beverage (i.e. beer) in Western countries contains folate, but Chinese liquor contains a high concentration of alcohol barely with folate content in northern China. Other possible reasons were poorer habits with lower intakes of folate, $\mathrm{B}_{12}$ and $\mathrm{B}_{6}$ during alcohol drinking. Moreover, alcohol consumption may interfere with the absorption and metabolism of folate ${ }^{(21)}$.

Physical activity has been associated with lower homocysteine levels in some population studies, especially among elderly subjects. Another study has found physical activity to be independently associated with lower plasma tHcy levels, when adjusting for the confounding effect of the MTHFR polymorphism, and plasma levels of both B vitamins and folate, as well as demographic and lifestyle parameters ${ }^{(22)}$. The present study also showed a decrease in tHcy levels in subjects with a higher level of physical activity. Perhaps physically active lifestyles could be a surrogate to healthier habits, associated with better nutrition and lower exposure to oxidative stress.

There are a few studies about the level of education as a determinant of HHCY, but results have been conflicting ${ }^{(23)}$. The present study showed that lower education level was associated with HHCY. There are two possible reasons. One reason is that subjects with higher education level are so well informed that they have abundant knowledge of health care. Another reason is that patients with high education level often obtain better economic conditions and nutritional status compared with those with low education level.

The MTHFR $677 \mathrm{C} \rightarrow \mathrm{T}$ polymorphism is one of the important genetic determinants of plasma tHcy in many studies. It has been reported that tHcy levels increase from 20 to $35 \%$ in subjects with the MTHFR 677-TT genotype than in subjects with the 677-CC genotype ${ }^{(24)}$. The effect of the MTHFR $677 \mathrm{C} \rightarrow \mathrm{T}$ variant on homocysteine concentrations was larger in low-folate regions such as China than in areas with folate fortification such as America and New Zealand ${ }^{(25)}$. In the present study, the MTHFR polymorphism is a strong determinant of plasma tHcy levels. Men or women with MTHFR 677TT and CT genotypes ( $v$. CC genotype) had higher tHcy concentrations. However, there were no significant interaction effects between the MTHFR C677T polymorphism and sex on the prevalence of HHCY, as has been described in previous studies $^{(26)}$. Most importantly, the frequency of the MTHFR 677TT genotype was $27.4 \%$, similar to the result found in a previous hypertensive adult study in different Chinese regions ${ }^{(8,27)}$, but higher than that reported in other developed countries (about 5-18\%) ${ }^{(28,29)}$.

Inverse associations between serum folate and tHcy concentrations have been reported in a previous study. Plasma tHcy level has been found to be inversely associated with serum folate level ${ }^{(30)}$. Folic acid fortification has been found to result in a significant decrease in circulating tHcy levels in the $\mathrm{USA}^{(13)}$. However, a previous report from a meta-analysis of twenty-six randomised trials has found that dietary supplementation with folic acid has no significant effects on cardiovascular events and stroke within 3 years ${ }^{(31)}$. In the present study, folate and its interaction with genotype were not associated with the prevalence of HHCY, as determined by the multivariate logistic regression analysis.

There are some limitations of the present study. First, the present study is a cross-sectional study, which does not allow for causal relationships among variables to be 
established. Therefore, it is necessary for us to conduct subsequent studies to confirm the hypotheses generated. Second, some confounding variables were not included in the present study, such as diet, mecobalamin intake, vitamin $\mathrm{B}_{6}$ and creatinine levels, which have been linked to plasma homocysteine levels.

In summary, we simultaneously evaluated the effect of demographic data, lifestyle, education level, a major genetic polymorphism and biochemical indicators on the prevalence of HHCY in a sample of healthy adults of Shaanxi Province. These data provide strong evidence for a high prevalence of HHCY and folate deficiency. Sex, smoking status, alcohol drinking, education level, physical activity and the MTHFR genotype were significant determinants. The concomitant change in lifestyle, including cessation of smoking and alcohol drinking, and improvement in physical activity levels may help decrease tHcy concentrations and reduce the risk of CVD in this population.

\section{Acknowledgements}

The present study was supported by the Shaanxi Province Association of Neurology and the National Natural Science Foundation of China (to X.-D. L., grant no. 81171236).

The authors' contributions are as follows: G. Z., Q.-H. J. and X.-P. X. designed the research; X.-D. L., B. G. and Q.-H. J. helped with the acquisition of the data; M. S. and Y.-Y. M. carried out the laboratory analysis of the data; X.-D. L., B. W. and $X$. X. contributed to the analysis and interpretation of the data; X.-D. L., G. Z. and Q.-H. J. wrote the paper; G. Z., Q.-H. J., $X$. X. and X.-P. X. contributed to the critical revision of the manuscript for important intellectual content; G. Z. and Q.-H. J. had primary responsibility for the final content. All authors read and approved the final manuscript.

None of the authors had any personal or financial conflict of interest.

\section{References}

1. Cui R, Moriyama Y, Koike KA, et al. (2008) Serum total homocysteine concentrations and risk of mortality from stroke and coronary heart disease in Japanese: The JACC study. Atherosclerosis 198, 412-418.

2. Veeranna V, Zalawadiya SK, Niraj A, et al. (2011) Homocysteine and reclassification of cardiovascular disease risk. J Am Coll Cardiol 58, 1025-1033.

3. Ganji V \& Kafai MR (2003) Demographic, health, lifestyle, and blood vitamin determinants of serum total homocysteine concentrations in the third National Health and Nutrition Examination Survey, 1988-1994. Am J Clin Nutr 77, $826-833$.

4. Homocysteine Lowering Trialists' Collaboration (2005) Dose-dependent effects of folic acid on blood concentrations of homocysteine: a meta-analysis of the randomized trials. Am J Clin Nutr 82, 806-812.

5. Till U, Rohl P, Jentsch A, et al. (2005) Decrease of carotid intima-media thickness in patients at risk to cerebral ischemia after supplementation with folic acid, vitamins $B_{6}$ and $\mathrm{B}_{12}$. Atherosclerosis 181, 131-135.
6. Harmon DL, Woodside JV, Yarnell JW, et al. (1996) The common 'thermolabile' variant of methylene tetrahydrofolate reductase is a major determinant of mild hyperhomocysteinaemia. QJM 89, 571-577.

7. Refsum H, Nurk E, Smith AD, et al. (2006) The Hordaland Homocysteine Study: a community-based study of homocysteine, its determinants, and associations with disease. J Nutr 136, 1731S-1740S.

8. Wang Y, Li X, Qin X, et al. (2012) Prevalence of hyperhomocysteinaemia and its major determinants in rural Chinese hypertensive patients aged 45-75 years. Br J Nutr 109, 1284-1293.

9. Hao L, Ma J, Zhu J, et al. (2007) High prevalence of hyperhomocysteinemia in Chinese adults is associated with low folate, vitamin $\mathrm{B}_{12}$, and vitamin $\mathrm{B}_{6}$ status. J Nutr $\mathbf{1 3 7}$, 407-413.

10. Yang W, Lu J, Weng J, et al. (2010) Prevalence of diabetes among men and women in China. $N$ Engl J Med 362, 1090-1101.

11. Tan Y, Sun X, Tang L, et al. (2003) Automated enzymatic assay for homocysteine. Clin Chem 49, 1029-1030.

12. Molloy AM \& Scott JM (1997) Microbiological assay for serum, plasma, and red cell folate using cryopreserved, microtiter plate method. Methods Enzymol 281, 43-53.

13. Ganji V \& Kafai MR (2006) Trends in serum folate, RBC folate, and circulating total homocysteine concentrations in the United States: analysis of data from National Health and Nutrition Examination Surveys, 1988-1994, 1999-2000, and 2001-2002. J Nutr 136, 153-158.

14. Selhub J, Jacques PF, Wilson PW, et al. (1993) Vitamin status and intake as primary determinants of homocysteinemia in an elderly population. JAMA 270, 2693-2698.

15. Hirsch S, de la Maza P, Barrera G, et al. (2002) The Chilean flour folic acid fortification program reduces serum homocysteine levels and masks vitamin B-12 deficiency in elderly people. J Nutr 132, 289-291.

16. Nygard O, Vollset SE, Refsum H, et al. (1995) Total plasma homocysteine and cardiovascular risk profile. The Hordaland Homocysteine Study. JAMA 274, 1526-1533.

17. Wouters MG, Moorrees MT, van der Mooren MJ, et al. (1995) Plasma homocysteine and menopausal status. Eur J Clin Invest 25, 801-805.

18. Haj MD, Ezzaher A, Neffati F, et al. (2011) Effect of cigarette smoking on plasma homocysteine concentrations. Clin Chem Lab Med 49, 479-483.

19. Heese P, Linnebank M, Semmler A, et al. (2012) Alterations of homocysteine serum levels during alcohol withdrawal are influenced by folate and riboflavin: results from the German Investigation on Neurobiology in Alcoholism (GINA). Alcohol Alcohol 47, 497-500.

20. Jacques PF, Bostom AG, Wilson PW, et al. (2001) Determinants of plasma total homocysteine concentration in the Framingham Offspring cohort. Am J Clin Nutr 73, 613-621.

21. Halsted CH, Villanueva JA, Devlin AM, et al. (2002) Metabolic interactions of alcohol and folate. J Nutr 132, 2367S-2372S.

22. Dankner R, Chetrit A, Dror GK, et al. (2007) Physical activity is inversely associated with total homocysteine levels, independent of C677T MTHFR genotype and plasma B vitamins. Age (Dordr) 29, 219-227.

23. Ganji V \& Kafai MR (2004) Serum total homocysteine concentration determinants in non-Hispanic White, nonHispanic Black, and Mexican-American populations of the United States. Ethn Dis 14, 476-482.

24. Yang QH, Botto LD, Gallagher M, et al. (2008) Prevalence and effects of gene-gene and gene-nutrient interactions 
on serum folate and serum total homocysteine concentrations in the United States: findings from the third National Health and Nutrition Examination Survey DNA Bank. Am J Clin Nutr 88, 232-246.

25. Holmes MV, Newcombe P, Hubacek JA, et al. (2011) Effect modification by population dietary folate on the association between MTHFR genotype, homocysteine, and stroke risk: a meta-analysis of genetic studies and randomised trials. Lancet 378, 584-594.

26. Papoutsakis C, Yiannakouris N, Manios Y, et al. (2006) The effect of MTHFR(C677T) genotype on plasma homocysteine concentrations in healthy children is influenced by gender. Eur J Clin Nutr 60, 155-162.

27. Qin X, Li J, Cui Y, et al. (2012) MTHFR C677T and MTR A2756G polymorphisms and the homocysteine lowering efficacy of different doses of folic acid in hypertensive Chinese adults. Nutr J 11, 2.
28. Gil-Prieto R, Hernandez V, Cano B, et al. (2009) Plasma homocysteine in adolescents depends on the interaction between methylenetetrahydrofolate reductase genotype, lipids and folate: a seroepidemiological study. Nutr Metab (Lond) 6, 39.

29. Hiraoka M (2004) Folate intake, serum folate, serum total homocysteine levels and methylenetetrahydrofolate reductase C677T polymorphism in young Japanese women. I Nutr Sci Vitaminol (Tokyo) 50, 238-245.

30. Ganji V \& Kafai MR (2009) Demographic, lifestyle, and health characteristics and serum B vitamin status are determinants of plasma total homocysteine concentration in the post-folic acid fortification period, 1999-2004. J Nutr 139, 345-352.

31. Yang HT, Lee M, Hong KS, et al. (2012) Efficacy of folic acid supplementation in cardiovascular disease prevention: an updated meta-analysis of randomized controlled trials. Eur J Intern Med 23, 745-754. 